\title{
Pre-participation assessment in young athletes: a state affair
}

\author{
Giorgio Galanti · Laura Stefani · Lorenzo Liverani • \\ Gian Franco Gensini
}

Received: 28 March 2012/Accepted: 26 May 2012/Published online: 12 June 2012

(C) SIMI 2012

One of the main goals for maintaining "wellness" is to promote and sustain physical activity as an "habitual practice". In both moderate and more vigorous forms, it normally generates numerous and well-known benefits in both young and not-so-young subjects [2]. More recently, sports activity, either in a competitive or non-competitive form, has started to involve a larger group of subjects from different age groups. Therefore, an accurate clinical evaluation in athletes is necessary. All the benefits obtained from sport activity are inevitably associated with several risks that can potentially induce various diseases, ranging from acute musculoskeletal injuries to sudden death [2, 3].

In this context, the Italian National Health Service has to deal with the protection of people who are going to start a competitive-elite sport activity. In fact, the National Health Service provides a medical check-up in order to allow all athletes to practice the type of physical activity best suited to maintain their well-being, to prevent avoidable diseases, and to maintain the highest level of safety during sport activity.

In this context, the pre-participation evaluation (PPE) represents the first step in the commencement of sport activity. This plan has also been adopted by European and

This text has been the object of an Invited Lecture given at the Royal College of Sports Medicine London by Professor Giorgio Galanti on 19 March 2010.

G. Galanti $\cdot$ L. Stefani $(\bowtie) \cdot$ L. Liverani

Sports Medicine Center, University of Florence,

Viale Morgagni 85, Florence, Italy

e-mail: laura.stefani@unifi.it

G. F. Gensini

Department of Medical and Surgical Critical Care,

University of Florence, Florence, Italy
American associations even if regular use and schemes have not yet been completely utilized.

According to ACSM, which is the main organization in US [4], athletes should undergo a complete examination before starting a seasonal competitive sport activity. In most states, this assessment is composed of a simple questionnaire, while only recently the electrocardiogram (ECG) has been introduced [5].

At present, the European PPE context is very dissimilar among countries and lacks a group of homogenous and standardized rules all leading to the same final objective.

For example, in Austria, pre-participation screening (PPS) may be performed from the age of 6 years under the guidance of dedicated-trained professionals; in Finland, where the risk of sudden death is estimated to be higher than elsewhere, PPS is required at least 6-8 weeks before starting sporting activities; in France, a general medical clearance to carry out physical activity is sufficient for "amateur sports", while in the case of "competitive sports", certification of the absence of physical contraindications is necessary; in The Netherlands, all citizens are currently submitted to a regular screening, and therefore a special program does not exist for all sports, as is the case of many other countries in Europe.

In the UK, in order to better monitor the impact of high level sport activity, this type of assessment is requested exclusively for elite athletes [6, 7].

Since 1982, in Italy, PPE, mandatory for competitive athletes, is officially under the supervision of the state. When an athlete is aged under 18 years, the National Health Service guarantees all the support required, and no additional financial contribution to obtain the eligibility is necessary.

The Italian PPE is composed of several recommended exams, in addition to clinical history and physical 
examination, in an attempt to detect significant clinical alterations and to assess the level of heart performance. Among them, an orthopedic evaluation, a lung functional examination by spirometry, an ECG, the cardiac maximal performance assessment by Step test or Harvard test, and also a urine analysis are included. Only for special kinds of sports, such as skiing or rally-cross, motor cycling, and skydiving, additional neurological examinations and audiometric check-ups are required.

All the examinations included in the PPE are established by the statements of the Italian National Olympic Committee (C.O.N.I). They are currently attempting to define the best age to start each sport. The authorization is usually given for 1 year, but in a few cases, its validity is occasionally extended to 2 years.

The PPE usually includes different examinations, while a "screening" normally incorporates just one. This is the main reason to consider a PPE evaluation not just a variety of "simply screening", but a more complex clinical evaluation.

So, even if in Italy, the application of the PPE is the first step with which to commence any sport activity, it is really a more extensive clinical approach, addressed not only to the evaluation of performance but also to maintain the wellness of young people.

The PPE is a program very different from the theoretic model of an effective "screening program", because it is not based on a free and voluntary assent to the program. The PPE is in fact strongly characterized by the compulsory nature of medical evaluation, which allows the athlete to obtain the license that authorizes her/him to practice a sport activity at a competitive level. Actually, an athlete could lie about the true nature of her/his health condition, and this behavior would seriously compromise the epidemiological evaluation.

From this point of view, the current and modern concept of the Italian PPE is therefore more complex, with clinical and social implications, all addressed to protect at the same time the wellness, along with inviolable human rights, and therefore overcoming the standard feature of the screening.

In conclusion, the PPE represents a global evaluation focusing on the health and the wellness of the young athletic population in addition to the evaluation of performance limited only to the kind and degree of sport practiced.

Moreover, the Italian PPE, in addition to a mean of clinical evaluation to obtain the "license" that authorizes a competitive sport practice, becomes an opportunity to gather statistical data on a large group of athletes, and thus to set up databases with epidemiological information on the state of health of the athletes and on their lifestyle.

\section{Discussion and conclusions}

In accordance with and observance of one's own ability and preferences, the Italian Constitution offers each citizen the right to undertake an activity or function that will contribute to the material and moral progress of society. Regarding competitive eligibility [8], Italian legislation includes a series of laws where the protection aspects in several sports activities are described.

The Italian Republic recognizes and guarantees inviolable human rights, for both individuals and social groups, following the expression of their traits, and consequently several laws regulate competitive eligibility [9, 10].

Some Italian laws have been also implemented to defend and protect special categories of subjects. The authorization to practice elite-competitive sports often requires an accurate analysis of prevention and treatment needs for these athletes.

Especially in the case of non-amateurs, sport activities are considered a stimulus towards improvement and progress.

Previous documents [8, 9] have highlighted exclusively the Italian PPE role as a screening devoted to the population practicing competitive sports; however, some aspects have reduced its relevance and applicability in other countries.

The low incidence of fatal acute cardiac events in competitive athletes [10], approximately 0.5-2.0 cases per 10,000 athletes per year, lower than in the general population, and the high cost/efficacy ratio of these procedures of screening actually represent the main limitations to propose the extension of this method to other countries. By comparing the Italian experience with other countries such as US [11-13] and European countries where the 12-lead ECG is compulsory to obtain eligibility, it is reasonable to think that the Italian PPE program is probably impractical as it would require considerable resources that are currently not available. The Italian experience itself [14-16] has demonstrated that the application over the last 30 years in the Veneto region of the ECG screening has determined an $89 \%$ absolute risk decrease of sudden cardiac death in competitive athletes, while the association of 12-lead ECG plus cardiovascular-focused history and physical examination may produce more additional benefits with a reasonable cost-effective ratio [17].

Especially in the case of young people, the PPE for physical activity is not currently aimed to disqualify or exclude athletes from competing, but rather, to help them maintain health and safety during training. This issue is now more relevant, considering a large and recently increasing cohort of overweight subjects among the competitive athletes. Any restriction from participation must be made based on the best medical objective evidence [13], 
and should be determined by keeping the cardiac and aerobic demands of the proposed activity in mind. An understanding of the strenuousness activity in relation to the sport practiced, and to its physical limitations, is crucial.

If the clearance is denied, recommendations for a correction prior to participation will be communicated, and a follow-up evaluation will be programmed. If acute illnesses or correctable conditions are resolved prior to the sport season, clearance will be given.

In conclusion, if on one hand the Italian PPE does not have the scientific feature of "screening" due to the compulsory nature of its procedures, on the other it offers a significant database on the state of health of the athletes. The introduction of the Sports Medicine check-up has drastically reduced the incidence of sudden death in the period dating from 1979 to 1997 , passing from 3.8/100.000 to $0.4 / 1000$, and aligning with the USA incidence [3].

The individual clinical evaluation represents, moreover, an important opportunity to understand better the habits and lifestyles of young people, and is therefore useful to help them avoid risky behaviors as well as to prevent 'dropping out' of physical and sport activity. As a consequence, some other general aspects beyond the cost-efficacy have to be considered in this context. The success of the Italian approach has led the European Society of Cardiology to adopt a common European protocol, including ECG as the centerpiece of cardiovascular screening [10], and the International Olympic Committee to recommend ECG screening for Olympic athletes. So, if the ECG plus cardiovascular focused history and physical examination is reasonable in cost, and is effective in saving lives, why does the Italian National Health Service not extend it globally to all young people, including the non-competitive sport practitioners? This in fact would be more reasonable, since it is not easy to distinguish between the two categories, especially where the work load and intensity of physical engagement are concerned.

Therefore, the aim of the PPE is not simply a score to identify people at high risk and to reduce the incidence of cardiovascular events among athletes, but, if globally addressed, a tool to produce positive criteria to allow sport activity in the largest part of the population investigated, and to mitigate the initial inevitable costs [17].

\section{Conflict of interest None.}

\section{References}

1. Haskell WL, Lee I-M, Pate RR, Powel KE, Blair SN, Franklin BA et al (2007) Physical activity and public health: updated recommendation for adults from the American College of Sports
Medicine and the American Heart Association. Med Sci Sports Exerc 39:1423-1434

2. Runyan DK (1983) The preparticipation examination of young athlete. Clin Pediatr 22:674-679

3. Leavitt MO (2008). Physical Activity Guidelines Advisory Committee Report To the Secretary of Health and Human Services US Department of Health and Human Services

4. Mac Auley D, Best TM (1976) Medical evaluation of athlete: a guide revised. In: Evidence-based sports medicine, 2nd edn. American Medical Association, Chicago, III

5. Maron BJ, Thompson PD, Ackerman MJ, Balady J, Berger S, Cohen D et al (2007) Recommendations and considerations related to pre-participation screening for cardiovascular abnormalities in competitive athletes: 2007 update: a scientific statement from the American Heart Association on Nutrition, Physical activity and Metabolism: endorsed by American College of Cardiology Foundation. Circulation 115:1643-455

6. Batt ME, Jacques R, Stone M (2004) Pre participation examination (screening): practical issues as determinant by sport: a United Kingdom perspective. Clin J Sport Med 14:178-182

7. Elston J, Stein K (2011) Public health implications of establishing national program to screen young athletes in UK. Br J Sports Med 45:576-582

8. Decree of the Italian Ministry of Health (1982) Norme per la tutela sanitaria dell'attivita' sportiva agonistica (rules concerning the medical protection of athletic activity). Gazzetta Ufficiale March 5:63

9. Comitato organizzativo cardiologico per l'idoneita ' allo sport (FMSI, SIC-Sport, SIC, ANCE, ANMCO) (1989) Protocolli cardiologici per il giudizio di idoneita‘ allo sport agonistico. G Ital Cardiol 19:250-272

10. Corrado D, Pelliccia A, Bjornstat HH, Vanhees L, Biffi A, Borjesson $M$ et al (2005) Cardiovascular pre-participation screening of young competitive athletes for prevention of sudden death: proposal for a common European protocol Consensus Statement of the Study Group of Sport Cardiology of the Working Group of Cardiac Rehabilitation and Exercise Physiology and the Working Group of Myocardial and Pericardial Diseases of the European Society of Cardiology. Eur Heart J 26:516-524

11. Maron BJ, Zipes PD (2005) Bethesda conference report: 36th Bethesda Conference Eligibility recommendation for competitive athletes with cardiovascular abnormalities. JACC 45:1322-1326

12. Corrado D, Basso C, Rizzoli G, Schiavon M, Thiene G (2003) Does sports activity enhance the risk of sudden death in adolescents and young adults? J Am Coll Cardiol 42:1959-1963

13. Maron BJ, Haas TS, Doerer DJ, Thompson PD, Hodges JS (2009) Comparison of US and Italian experiences with sudden cardiac deaths in young competitive athletes, and implications for preparticipation screening strategies. Am J Cardiol 104:276-280

14. Pelliccia A, Maron BJ (1995) Pre-participation cardiovascular evaluation of the competitive athlete: perspectives from the 30-year Italian experience. Am J Cardiol 75:827-829

15. Pelliccia A, DiPaolo F, De Luca R, Buccolieri C, Maron BJ (2001) Efficacy of pre-participation screening for the detection of cardiovascular abnormalities at risk of sudden death in competitive athletes: the Italian experience. J Am Coll Cardiol 37:151A (Abstract)

16. Wigfield K, Matheson GO, Meeuwisse GH (2004) Pre participation evaluation: an evidence based review. Clin J Sport Med 14:109-122

17. Wheeler MT, Heidenreich PA, Froelicher FV, Hlatky MA, Ashley EA (2010) Cost-effectiveness of preparticipation screening for prevention of sudden cardiac death in young athletes. Ann Intern Med 152:276-286 